\title{
EFECTO DE LAS ENMIENDAS LÍQUIDAS EN UN ULTISOL CULTIVADO CON SORGO ${ }^{1}$
}

\author{
Manuel E.Camacho ${ }^{2}$, Gilberto Cabalceta-Aguilar ${ }^{2}$, Eloy Molina-Rojas $^{2}$
}

\section{RESUMEN}

Efecto de las enmiendas líquidas en un Ultisol cultivado con sorgo. El objetivo de este trabajo fue evaluar el efecto de la aplicación de enmiendas líquidas en un Ultisol cultivado con sorgo. Esta investigación se realizó entre agosto y noviembre del 2011 en el Centro de Investigaciones Agronómicas, San José, Costa Rica. En potes de $800 \mathrm{ml}$ de un Ultisol sembrado con sorgo, se aplicaron los siguientes tratamientos: testigo sin cal, carbonato de calcio en dosis de 10 y 20 1/ha, óxido de magnesio en dosis de 10 y 20 1/ha, carbonato de calcio + óxido de magnesio en dosis de $5+5 \mathrm{y}$ $10+10$ 1/ha, respectivamente. Las plantas fueron cosechadas a las seis semanas, a las que se les determinó área foliar, peso seco y fresco de biomasa aérea y radicular, absorción de nutrimentos y características químicas del suelo. Los tratamientos de carbonato de calcio y en mezcla con óxido de magnesio obtuvieron los mejores valores de área foliar y los valores más altos de peso fresco y seco tanto para raíz como parte aérea del sorgo. No hubo diferencias significativas entre tratamientos de cal líquida pero si con respecto al testigo sin cal con las variables de peso de biomasa. El carbonato de calcio líquido aumentó la absorción de Ca de forma significativa, y el tratamiento de carbonato + óxido en dosis de 10 1/ha presentó la mayor absorción de Mg. Todos los tratamientos de enmiendas causaron un mejoramiento de la fertilidad del suelo, siendo el más destacado de ellos el tratamiento de óxido de magnesio en dosis de 20 1/ha, que disminuyó la acidez intercambiable de 9,02 a 0,36 cmol(+)/1, el porcentaje de saturación de acidez bajó de 95 a 3,3\% y aumentó el pH de 5,0 a 5,7. Se concluyó que las enmiendas líquidas tuvieron efecto positivo sobre el cultivo indicador y la fertilidad del suelo.

Palabras claves: acidez intercambiable, encalado, área foliar.

\begin{abstract}
Effect of liquid liming on sorghum growth in an Ultisol. The aim of this study was to evaluate the effects of the application of liquid lime on sorghum growth in an Ultisol. This research was conducted between August and November, 2011 at the Agricultural Research Center, San José, Costa Rica. In an Ultisol planted with sorghum, in pots of $800 \mathrm{ml}$, the following treatments where applied: control without lime, calcium carbonate at doses of 10 and $20 \mathrm{l} /$ ha, magnesium oxide at doses of 10 and 20 1/ha, calcium carbonate + magnesium oxide at doses of $5+5$ and $10+$ 10 1/ha, respectively. Six weeks after planting, sorghum was harvested, measuring leaf area, dry and fresh weight of the aerial and root biomass, nutrient absorption and the soil chemical characteristics. Treatments using calcium carbonate and calcium carbonate + magnesium oxide obtained the best values of leaf area and the higher weight of the aerial and root biomass of sorghum. Even though there were no significant differences between liquid lime treatments, there were regarding control without lime and weight biomass variables. Liquid calcium carbonate significantly increased $\mathrm{Ca}$ absorption, and the calcium carbonate + magnesium oxide treatment at doses of $10 \mathrm{l} / \mathrm{h}$ showed the highest $\mathrm{Mg}$ absorption. All amendment treatments caused an improvement of the soil fertility, the most notable being the application of $20 \mathrm{l} / \mathrm{ha}$ of magnesium oxide that dropped the exchangeable acidity from 9.02 to $0.36 \mathrm{cmol}(+) / 1$, acidity saturation dropped from 95 to $3.3 \%$, and $\mathrm{pH}$ increased from 5 to 5.7. It was concluded that the liquid liming amendments had a positive effect over the crop and the soil fertility.
\end{abstract}

Keywords: exchangeable acidity, liming, leaf area.

1 Recibido: 27 agosto, 2014. Aceptado: 13 de octubre, 2014. Este trabajo forma parte del proyecto VI 733-B0-137 de la Universidad de Costa Rica. San José, Costa Rica.

2 Universidad de Costa Rica, Centro de Investigaciones Agronómicas. San Pedro de Montes de Oca, San José, Costa Rica. manuel. camacho87@gmail.com, gilberto.cabalceta@ucr.ac.cr, eloy.molina@ucr.ac.cr 


\section{INTRODUCCIÓN}

La acidez del suelo se origina por la presencia de iones como el aluminio e hidrógeno que contribuyen a reducir el $\mathrm{pH}$, afectando tanto las características químicas como biológicas del suelo, reduciendo el crecimiento de las plantas y causando la disminución de la disponibilidad de algunos nutrimentos como $\mathrm{Ca}$, $\mathrm{Mg}, \mathrm{P}$ y K. Esto a su vez favorece la acumulación de elementos tóxicos para las plantas como el $\mathrm{Al}$ y el $\mathrm{Mn}$ (Bertsch, 1995; Alvarado et al., 2010).

Esta condición de suelo se genera por múltiples causas, desde la génesis del suelo hasta el manejo que se le de al mismo. La meteorización intensiva que a través de millones de años se ve sometido el suelo, y los procesos de pérdida de bases por lixiviación o por extracción de cultivos, se consideran como causas principales de la acidez de los suelos en regiones tropicales (Molina, 1998). Otro proceso involucrado, la hidrólisis del $\mathrm{Al}^{3+}$, es causante de producir grandes cantidades de iones $\mathrm{H}^{+}$en el sistema suelo, generando condiciones ácidas perjudiciales para el sistema radical (Alvarado y Fallas, 2004).

El sistema radical de las plantas constituye el mecanismo de absorción de agua y minerales presentes en el suelo por parte de las plantas; sus características están condicionadas por la genética de la especie, pero también por las condiciones edáficas, tanto físicas como químicas (Salisbury y Ross, 1999). El crecimiento de las raíces se ve favorecido generalmente en condiciones ligeramente ácidas, $\mathrm{pH}$ de 5,5 a 6,5 (Taiz y Zeiger, 2002). Sin embargo, la acidez en el suelo afecta el crecimiento de las raíces y disminuye en forma indirecta la capacidad de la planta para absorber agua. Estas condiciones tienen una relación entre el contenido de $\mathrm{Ca}$ y $\mathrm{Al}$ intercambiable, donde incrementos en el contenido de $\mathrm{Ca}$ y una reducción del $\mathrm{Al}$ intercambiable desarrollan mejores condiciones para el crecimiento del sistema radical (Kamprath, 1984; Alvarado et al., 2010).

El Al está muy asociado con problemas de acidez en el suelo, se considera como uno de los principales agentes involucrados en la reducción del crecimiento de las plantas, debido al efecto fitotóxico en ciertas especies que son poco tolerantes a dicho elemento (Brown et al., 2008). Este elemento es abundante en los suelos con condiciones tropicales, debido a la mineralogía de arcillas caoliníticas (1:1) y sesquióxidos de Fe y Al que se presentan en estos suelos (Salas et al.,
2002), los que generalmente poseen un $\mathrm{pH}$ bajo, lo cual potencializa la toxicidad del $\mathrm{Al}$ (Brown et al., 2008).

Los Ultisoles son suelos que se originan por el efecto prolongado de factores climáticos, principalmente alta precipitación, que forman suelos con bajos contenidos de bases y la acumulación de arcilla iluviada en el sub-suelo. Estos presentan buenas propiedades físicas, a pesar de ser muy arcillosos, su grado de fertilidad por lo general es baja, debido a bajos contenidos de $\mathrm{Ca}$ y $\mathrm{Mg}$, presencia de toxicidad de Al y baja disponibilidad de $\mathrm{P}$ (Bertsch, 1995).

En los Ultisoles, el Al constituye un problema muy serio, cuya principal repercusión recae en el retraso del crecimiento y desarrollo de los cultivos, específicamente provocando condiciones adversas que afectan la raíz (Molina y Rojas, 2005). La saturación alta de acidez propicia baja disponibilidad de P y K (Molina y Rojas, 2005), disminución en la absorción de $\mathrm{Ca}, \mathrm{Mg}$ y $\mathrm{N}$, así como un incremento de $\mathrm{Al}$ y $\mathrm{Mn}$ en cantidades perjudiciales para el desarrollo de la planta (Espinosa y Molina, 1999; Molina, 2001). Los Ultisoles son el grupo de suelos predominantes en Costa Rica, con un 56,67\% del territorio nacional, seguido por los Andisoles (16,68\%), Inceptisoles $(13,13 \%)$ y Alfisoles $(5,77 \%)$. Estos órdenes podrían tener problemas de acidez, principalmente los Ultisoles (Mata et al., 2012).

El manejo de la acidez del suelo constituye un proceso integral, debido a que la acidez involucra muchos factores, tanto químicos como físicos (Molina, 2001). El encalado constituye la forma más simplificada y efectiva de corregir este problema. Se basa en la aplicación de sales básicas que neutralizan la acidez causada por el $\mathrm{Al}$ e $\mathrm{H}$ presente en el suelo; sin embargo, se debe considerar aspectos importantes como la fuente del material de enmienda, la época, la dosis y el método de aplicación (Molina, 1998). Esta práctica estimula el crecimiento de la planta al reducir toxicidades del $\mathrm{Al}$ y $\mathrm{Mn}$, e incrementa la disponibilidad de nutrimentos como el Ca, N y Mo (Curtin y Syers, 2002). Además, disminuye la lixiviación de minerales presentes en la solución del suelo, debido a que se incrementa el $\mathrm{pH}$, reduciendo la solubilidad de los mismos (Taiz y Zeiger, 2002).

En la actualidad existen en el mercado muchos tipos de materiales que se emplean como enmiendas al suelo para corregir estos problemas de acidez. Algunos ejemplos son los carbonatos de $\mathrm{Ca}$ y $\mathrm{Mg}$, dentro de los cuales destacan la cal agrícola $\left(\mathrm{CaCO}_{3}\right)$, que es la 
enmienda más comúnmente utilizada (Molina, 1998). Otras son los óxidos e hidróxidos de Ca que provienen de procesos físicos de transformación de la roca caliza; y la cal dolomita $\left(\mathrm{CaMg}\left(\mathrm{CO}_{3}\right)_{2}\right)$, la cual se emplea cuando el nivel de $\mathrm{Mg}$ es bajo en el suelo. Además, se debe considerar la calidad de los materiales, expresada como el poder relativo de neutralización total (PRNT), el cual se obtiene en función de la granulometría y el componente químico o pureza química de un material (Alcarde, 1992; Molina, 2001). La fineza de las partículas individuales de la cal determina su velocidad de reacción; conforme se reduce el tamaño de la partícula de cualquier material, aumenta su área o superficie de contacto. Entre más fino el material, mayor es su poder neutralizante de acidez y más rápido es su efecto sobre el $\mathrm{Al}$ intercambiable y el pH del suelo (Espinosa y Molina, 1999).

Generalmente se utilizan enmiendas sólidas para el control de la acidez del suelo, pero también puede ser aplicada en forma líquida como una suspensión con el uso de algunos coadyuvantes como las arcillas atapulgita y bentonita, $\mathrm{y}$ un agente dispersante (Barber, 1984, Peters et al., 1996).

El beneficio de las enmiendas líquidas en el suelo se ha atribuido a su alta fineza, por ser productos de malla 625 mesh, que permite una reacción química en el suelo muy fuerte y rápida, de manera similar a como reaccionaría un óxido de calcio (Valerio y Molina, 2012).

Las enmiendas líquidas son una alternativa para el control de la acidez del suelo en situaciones donde no es posible la aplicación de productos sólidos o se dificulta su aplicación, como en el caso de cultivos ya sembrados, de crecimiento muy rápido donde se requiere una respuesta muy rápida de la enmienda, en cultivos sembrados con mínima labranza donde no puede incorporarse enmiendas sólidas, en cultivos con fertirriego donde la enmienda líquida puede ser inyectada en el sistema de riego, y en terrenos alquilados donde los agricultores no desean hacer una alta inversión en enmiendas debido a que tienen que devolver la tierra. Por este motivo, y debido a que un gran porcentaje de los cultivos a nivel nacional se desarrollan en suelos con problemas de acidez, y a la problemática que conlleva esta condición de suelo en la fisiología de la absorción de los nutrimentos por parte de las plantas, el presente trabajo tuvo como objetivo evaluar el efecto de la aplicación de enmiendas líquidas sobre un Ultisol cultivado con sorgo.

\section{MATERIALES Y MÉTODOS}

Este estudio se realizó entre los meses de agosto y noviembre del 2011 en el invernadero del Laboratorio de Suelos y Foliares del Centro de Investigaciones Agronómicas, Sabanilla, San José, Costa Rica. Se tomó aproximadamente $40 \mathrm{~kg}$ de suelo de los primeros $20 \mathrm{~cm}$ de profundidad, en un Ultisol de $\mathrm{pH}<5,5 \mathrm{y}$ acidez intercambiable $>9,0 \mathrm{cmol}(+) / 1$, ubicado en La Legua, Aserrí, San José. El suelo se clasificó como un Typic Rhodustults, con el horizonte A muy erosionado y con un sitio con el horizonte $\mathrm{B}_{\mathrm{t}}$ (argílico) completamente expuesto, lo que explica su alta acidez intercambiable. Se desmenuzó manualmente y se secó al aire por un período mínimo de 48 horas. Posteriormente, se pasó a través de un tamiz de $2 \mathrm{~mm}$ y se mezcló para obtener el mayor grado de homogeneidad posible en sus características químicas y físicas.

Las enmiendas a utilizar fueron las siguientes: carbonato de calcio, óxido de magnesio y una mezcla de $50 \%$ de cada una de ellas, todas en presentación líquida. El carbonato de calcio es una enmienda líquida de reciente introducción, y presenta $56 \%$ de $\mathrm{CaO}$, en forma de carbonato de calcio micronizado de malla 625 mesh, grado farmacéutico ( $<2$ micras), un $\mathrm{pH}$ entre 8,5 y 10 , y una densidad entre 1,65 y $1,69 \mathrm{~g} / \mathrm{cm}^{3}$ (es un líquido pesado). Contiene además $6,9 \%$ de $\mathrm{N}$ en forma de urea, lo que aparentemente favorecería una mejor respuesta a su aplicación. Presenta un coadyuvante que se llama goma Xanthana que permite la suspensión del material en agua. Al comparar el tamaño de partículas de este material con la superficie específica que posee la montmorillonita $\left(600-800 \mathrm{~m}^{2} / \mathrm{g}\right)$, se obtiene que un litro de carbonato de calcio líquido (560 g CaO) tiene $392000 \mathrm{~m}^{2}(560$ g x $700 \mathrm{~m}^{2}$ ). La dosis recomendada es de 8-10 1/ha. El óxido de magnesio es una enmienda líquida que utiliza la misma goma Xanthana como coadyuvante para mantener el producto en suspensión, y contiene $48 \%$ de $\mathrm{MgO}$ en forma de óxido, un $\mathrm{pH}$ entre 9 y 11,5 , y una densidad específica de $1,45 \mathrm{~g} / \mathrm{cm}^{3}$. El tamaño promedio de partículas es de 0,4 micras que corresponden con una malla mayor a 2500 mesh $(0,005 \mathrm{~mm})$.

Se realizó un análisis químico completo del suelo seleccionado para el estudio (Cuadro 1), determinando el $\mathrm{pH}$ en agua, $\mathrm{Ca}, \mathrm{Mg}$ y acidez intercambiable extraíble en $\mathrm{KCl} 1 \mathrm{~N}$; contenidos de $\mathrm{P}, \mathrm{K}, \mathrm{Fe}, \mathrm{Cu}, \mathrm{Zn}$, y Mn extraíble en Olsen Modificado, utilizando las 
Cuadro 1. Características químicas del suelo empleado. Laboratorio de Suelos del Centro de Investigaciones Agronómicas, Universidad de Costa Rica. San José, Costa Rica. 2011.

Table 1. Chemical characteristics of the soil used. Agricultural Research Center, University of Costa Rica. San José, Costa Rica. 2011.

\begin{tabular}{|c|c|c|c|c|c|c|c|c|c|c|c|}
\hline \multirow{2}{*}{$\begin{array}{c}\mathbf{p H} \\
\left(\mathbf{H}_{2} \mathbf{O}\right)\end{array}$} & Acidez* & $\mathbf{C a}$ & Mg & $\mathbf{K}$ & CICE & \multirow{2}{*}{$\begin{array}{c}\mathbf{S A}^{* *} \\
\%\end{array}$} & $\mathbf{P}$ & Zn & $\mathrm{Cu}$ & $\mathrm{Fe}$ & Mn \\
\hline & \multicolumn{5}{|c|}{$\operatorname{cmol}(+) / l$} & & \multicolumn{5}{|c|}{ mg/l } \\
\hline 5,0 & 9,02 & 0,35 & 0,06 & 0,10 & 9,53 & 95 & ND & 1,6 & 2 & 35 & 5 \\
\hline
\end{tabular}

*Acidez intercambiable / Exchangeable acidity.

** Porcentaje de saturación de acidez intercambiable / Percentage of Exchange acidity saturation. ND: no detectado / ND: non detected.

metodologías del Laboratorio de Suelos del Centro de Investigaciones Agronómicas, de acuerdo con lo propuesto por Díaz-Romeu y Hunter (1978).

El suelo se colocó en potes de $800 \mathrm{ml}$ y los tratamientos se aplicaron según la dosis indicada para cada uno. A las enmiendas líquidas se les realizó una dilución de cada dosis en un volumen de $400 \mathrm{ml}$ de agua. El tratamiento consistió en aplicar una lámina de riego de $400 \mathrm{ml}$ de agua, en la cual se adicionó un volumen de la enmienda según el tratamiento; el carbonato de calcio en dosis de 10 1/ha se agregó $2 \mathrm{ml}$ diluidos en $400 \mathrm{ml}$ de agua, y $4 \mathrm{ml}$ diluidos en $400 \mathrm{ml}$ de agua para la dosis 20 1/ha. El óxido de magnesio, debido a su consistencia pastosa, se diluyó en una relación 1:1, de esta dilución se tomó 4 y $8 \mathrm{ml}$ diluidos en $400 \mathrm{ml}$ de agua para una dosis de 10 y $20 \mathrm{l} / \mathrm{ha}$, respectivamente. Para los tratamientos de la mezcla de carbonato de calcio + óxido de magnesio, se adicionó la mitad de los $\mathrm{ml}$ necesarios para las dosis de 10 y 20 1/ha mencionados anteriormente, para ambas enmiendas. No existe un método para calcular dosis de enmiendas líquidas con base en una fórmula como ocurre con las sólidas, por lo que en este caso se utilizaron las dosis recomendadas por el fabricante de 10 a 20 1/ha diluidos en el volumen mencionado anteriormente.

Hubo respuesta significativa en rendimiento de arroz en granza a la aplicación de carbonato de calcio en dosis de $81 /$ ha, asperjado sobre las plantas y el suelo a los ocho días de germinado el cultivo (Valerio y Molina, 2012), por lo anterior, se decidió sembrar el sorgo una semana después de aplicados los tratamientos. Para poder evaluar el efecto de corto plazo que se ha indicado que poseen las enmiendas líquidas para mejorar algunos de los parámetros de la fertilidad del suelo, se tomaron muestras en cada tratamiento a los 4, 6, 10,18, 25 y 44 días después de la aplicación de enmiendas, para analizar el contenido de la acidez intercambiable y $\mathrm{pH}$ en agua, para todo lo anterior se utilizaron potes paralelos. La justificación de hacer estos muestreos radica en el hecho de que los materiales evaluados se consideraron de gran reactividad debido a su elevada fineza y su estado líquido, que teóricamente causarían cambios químicos de corto plazo en el suelo, por lo que el objetivo de realizar estos muestreos fue determinar si efectivamente se cumple esta hipótesis.

Se sembraron veinte semillas de sorgo por pote. El riego del cultivo se realizó por capilaridad, mediante un recipiente de plástico en el cual se introdujo el pote con agujeros en el fondo. El recipiente contenía agua desionizada con $50 \mathrm{mg} / \mathrm{l}$ de $\mathrm{N}$ cuya fuente era $\mathrm{NH}_{4} \mathrm{NO}_{3}$, de acuerdo con lo propuesto por Cabalceta y Molina (2006) y Molina y Bornemisza (2006). Todos los potes recibieron la solución de $50 \mathrm{mg} / \mathrm{l}$ de $\mathrm{N}$ de forma permanente durante el tiempo del experimento. Aunque el carbonato de calcio líquido tenía nitrógeno proveniente de la urea, se consideró que no tuvo efecto en el crecimiento del sorgo puesto que todos los potes recibieron una dosis de $\mathrm{N}$ de más de doce veces superior a la que aportó la cal, mediante el suministro continuo en el riego de nitrato de amonio durante los 35 días que tardó la prueba con las plantas.

Se realizó un análisis químico de suelos completo al final del experimento ( 45 días después de la siembra de sorgo) en todas las repeticiones y tratamientos.

Se hizo un análisis químico de la biomasa seca de la parte aérea de la planta para la determinación de $\mathrm{N}, \mathrm{P}, \mathrm{Ca}, \mathrm{Mg}, \mathrm{K}, \mathrm{S}, \mathrm{Fe}, \mathrm{Cu}, \mathrm{Zn}, \mathrm{Mn}, \mathrm{y} \mathrm{B}$, utilizando el método de digestión del material vegetal con $\mathrm{HNO}_{3}$, y analizando los nutrimentos por medio de espectroscopía de emisión atómica (ICP), a través de los procedimientos establecidos por Díaz-Romeu 
y Hunter (1978). Con los resultados de este análisis y los datos de peso seco se calculó la absorción de nutrimentos en la biomasa aérea.

\section{Diseño experimental}

El ensayo se realizó en un invernadero con un diseño experimental irrestricto al azar, con siete tratamientos y cuatro repeticiones para cada uno.

Los tratamientos que se aplicaron fueron los siguientes:

- Testigo sin enmienda (T).

- Carbonato de calcio en dosis de 10 y 20 1/ha (C10 y C20 respectivamente).

- Óxido de magnesio en dosis de 10 y $20 \mathrm{l} / \mathrm{ha}$ (Mg 10 y Mg 20 respectivamente).

- Carbonato de calcio + óxido de magnesio mezclados en dosis de $5+5$ y $10+10$ l/ha de cada uno $(\mathrm{C} 5+\mathrm{Mg} 5$ y C10 $+\mathrm{Mg} 10$ respectivamente).

\section{Variables fisiológicas}

Las variables evaluadas de acuerdo a lo propuesto por Guevara y Jiménez (1998) fueron las siguientes: a) área foliar, se realizó mediante el uso del software WinFolia ${ }^{\circledR}$, determinando el área foliar mediante un escáner, sobre el cual se colocó la parte aérea de las plantas que conformaban cada repetición, b) peso de biomasa fresca y seca al final para cada una de las partes morfológicas de las plantas (raíz, tallo y lámina foliar), el cual se realizó mediante una balanza granataria, con una precisión de dos decimales. El peso seco se determinó después de secar las muestras en estufa a $70{ }^{\circ} \mathrm{C}$ durante 48 horas, y c) absorción de nutrimentos, calculado a partir del análisis foliar del cultivo y el peso seco de la parte aérea.

\section{Variables del suelo}

Se evaluaron también las condiciones químicas del suelo al inicio y al final del ensayo, mediante análisis químico de suelo, los cuales se realizaron en el Laboratorio de Suelos y Foliares del Centro de Investigaciones Agronómicas, cuya metodología estuvo conforme con lo propuesto por Díaz-Romeu y Hunter (1978). Las variables a evaluar fueron: contenido de nutrimentos, $\mathrm{pH}$, acidez intercambiable y porcentaje de saturación de acidez en el suelo.

\section{Análisis estadístico}

Mediante el uso del paquete estadístico STATISTICA $^{\circledR}$ se realizó el análisis de varianza "ANDEVA" para todas las variables, y se utilizó la prueba de Tukey para separar las medias en las variables que presentaron diferencias mínimas significativas $(\mathrm{p}<0,05)$.

\section{RESULTADOS Y DISCUSIÓN}

\section{Efecto de las enmiendas en el área foliar}

Los tratamientos de carbonato de calcio líquido y el de carbonato de calcio 10 1/ha + óxido de magnesio 10 1/ha (Ca $10+\mathrm{Mg}$ 10) presentaron los valores de área foliar más elevados (Figura 1). Se observaron diferencias significativas con respecto al testigo sin cal. Los tratamientos de óxido de magnesio líquido no presentaron diferencias estadísticas con el testigo, y mostraron los valores más bajos del área foliar, probablemente porque afectaron de forma negativa el crecimiento de las plantas, ya que según Molina (1998) el óxido de magnesio tiene más del doble de poder neutralizante de la acidez del suelo que el carbonato de calcio, por lo que es capaz de subir el $\mathrm{pH}$ y reducir el $\mathrm{Al}$ intercambiable en mayor grado que el carbonato, y en causar un bloqueo en la absorción de calcio. Además, el suelo tenía contenidos muy bajos de Ca (Cuadro 1) y la aplicación de óxido de magnesio probablemente afectó en forma negativa la absorción de Ca y por ende, redujo el área foliar. Los tratamientos de óxido de magnesio fueron estadísticamente similares al testigo sin cal, lo que reforzó más la observación de un posible antagonismo que afectó la absorción de Ca en la planta.

Al respecto Ritche y Snuffer (2002), encontraron un incremento en el rendimiento del forraje de Dactylis glomerata L. y Festuca arundinacea Schreb, sometidas a tratamientos de enmiendas como dolomita y fosfoyeso. Tang et al. (2001, 2003) encontraron incrementos del $50 \%$ y $60 \%$ en el rendimiento de cultivos como cebada y trigo, bajo tratamientos de enmienda, en suelos con condiciones de alta saturación de acidez. El incremento del crecimiento vegetal asociado a aplicación de enmiendas no se relaciona sólo con la neutralización de iones tóxicos, sino 


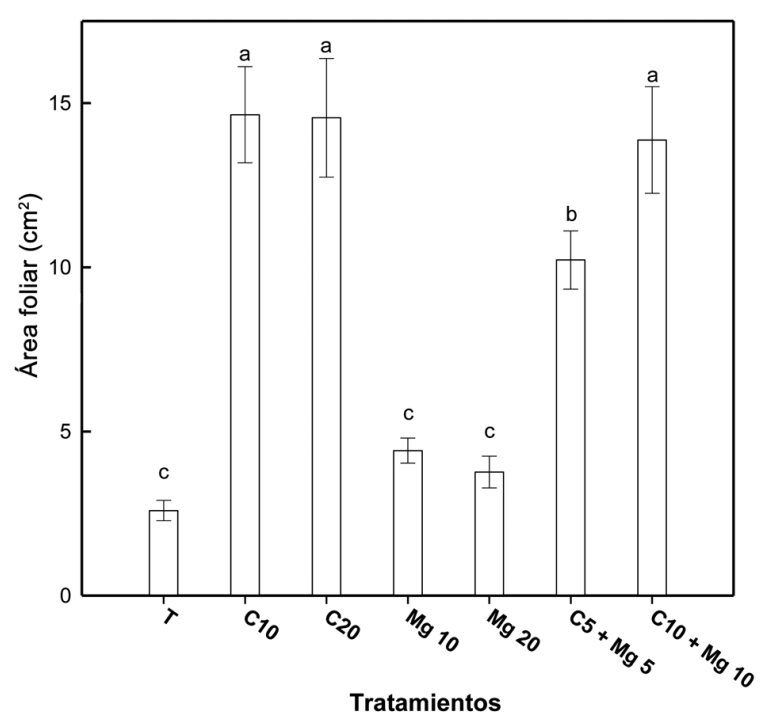

Figura 1. Efecto de la aplicación de enmiendas líquidas en el área foliar del sorgo sembrado en potes con suelo Ultisol. San Pedro de Montes de Oca, San José, Costa Rica. 2011.

$\mathrm{T}=$ testigo sin enmienda; carbonato de calcio en dosis de 10 y 20 l/ha (C10 y C20 respectivamente), óxido de magnesio en dosis de 10 y 20 l/ha (Mg10 y $\mathrm{Mg} 20$ respectivamente), carbonato de calcio + óxido de magnesio mezclados en dosis de $5+5$ y $10+10$ 1/ha de cada uno (C5 + Mg5 y C $10+\mathrm{Mg} 10$ respectivamente). Barras de error con base en error estándar. Letras diferentes implican diferencias estadísticas entre tratamientos, según la prueba de Tukey $(\mathrm{p}<0,05)$.

Figure 1. Effect of liquid liming amendments on leaf area of sorghum planted in pots with an Ultisol. San Pedro, Montes de Oca, San José, Costa Rica. 2011. $\mathrm{T}=$ control without lime; calcium carbonate at 10 and 20 1/ha doses (C10 and $\mathrm{C} 20$ respectively), magnesium oxide at 10 y $20 \mathrm{l} / \mathrm{ha}$ doses $(\mathrm{Mg} 10$ and $\mathrm{Mg} 20$, respectively), calcium carbonate + magnesium oxide mixed at $5+5$ and $10+10$ 1/ha doses of each one (C5 + Mg5 and C10 $+\mathrm{Mg} 10$, respectively). Error bars as standard error. Different letters mean statistical differences between treatments (Tukey test, $\mathrm{p}<0.05$ ).

también con el incremento en la disponibilidad de $\mathrm{Ca}$, N y Mo (Edmeades y Ridley, 2003).

\section{Efecto de las enmiendas en la biomasa aérea}

Los tratamientos de carbonato de calcio solo o con óxido de magnesio, resultaron con valores más elevados tanto para biomasa fresca y seca, y no hubo diferencia estadística entre ellos (Figura 2).

Los tratamientos de óxido de magnesio presentaron los valores más bajos, pero estadísticamente superiores al testigo en peso fresco. Para el peso seco se presentó un comportamiento similar a excepción del tratamiento de $\mathrm{Mg}$ 20, el cual estadísticamente fue similar al testigo. Como se indicó anteriormente, es probable que el óxido de magnesio causara una severa deficiencia de $\mathrm{Ca}$ debido al antagonismo existente entre ellos y que a su vez se presentara una leve intoxicación en las plantas debido a su fuerte poder neutralizante de acidez en comparación con el carbonato de calcio (Molina, 1998). Los resultados también mostraron que el Ca tuvo mayor efecto en la producción de biomasa que el Mg, y que la aplicación de solo Ca causó el mismo efecto que el uso de $\mathrm{Ca}+\mathrm{Mg}$, mostrando la gran importancia que tiene la deficiencia de $\mathrm{Ca}$ en muchos suelos fuertemente ácidos como el del presente estudio.

Los resultados anteriores concuerdan con Soratto y Crusciol (2008), quienes encontraron un incremento en el contenido de materia seca del arroz como consecuencia de la aplicación de diferentes dosis de cal dolomita. Lo mismo reportaron Tang et al. (2001, 2003) en sus investigaciones donde mostraron un incremento de materia seca en la parte aérea debido a la aplicación de enmienda. Valerio y Molina (2012) encontraron respuesta significativa en el rendimiento de arroz con la aplicación del carbonato de calcio líquido en dosis de 8 1/ha en un suelo ácido de Los Chiles, Costa Rica.

La acidez del suelo puede presentar una correlación negativa con la biomasa vegetal; así se mostró en pasto Kikuyo (Kikuyo chloa clandestinum anteriormente Pennisetum clandestinum); donde también se encontró que el Al causó una disminución en la calidad nutricional del pasto forrajero (Sidari et al., 2004).

También se reportó una respuesta al encalado por parte de especies maderables nativas de la sabana brasileña, con un incremento en la producción de materia seca total (Furtini et al., 1999). Tang et al. (2003), encontraron que la aplicación de cal aumentó la biomasa en la parte aérea del trigo de 45 a $70 \%$.

\section{Efecto de las enmiendas en la biomasa de raíces}

Los tratamientos de carbonato de calcio solo o con óxido de magnesio mostraron los valores más altos de peso fresco de raíces, y hubo diferencias altamente 


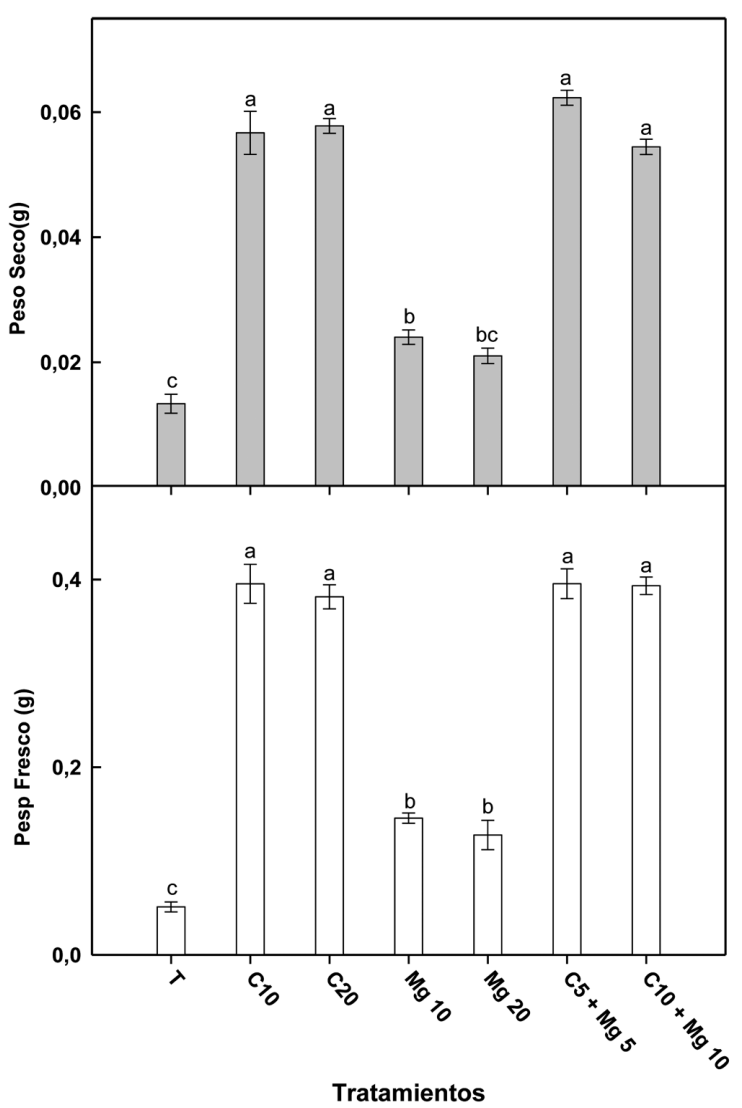

Figura 2. Efecto de la aplicación de enmiendas líquidas sobre la biomasa aérea del sorgo sembrado en potes con suelo Ultisol. San Pedro de Montes de Oca, San José, Costa Rica. 2011.

$\mathrm{T}=$ testigo sin enmienda; carbonato de calcio en dosis de 10 y 20 1/ha (C10 y C20 respectivamente), óxido de magnesio en dosis de 10 y 20 l/ha $(\mathrm{Mg} 10$ y $\mathrm{Mg} 20$ respectivamente), Carbonato de calcio + oxido de magnesio mezclados en dosis de $5+5 \mathrm{y}$ $10+10$ 1/ha de cada uno $(\mathrm{C} 5+\mathrm{Mg} 5$ y C10 + Mg10 respectivamente). Barras de error con base en error estándar. Letras diferentes implican diferencias estadísticas entre tratamientos, según la prueba de Tukey $(\mathrm{p}<0,05)$.

Figure 2. Effect of liquid liming amendments on aerial biomass of sorghum planted in pots with an Ultisol. San Pedro, Montes de Oca, San José, Costa Rica. 2011. $\mathrm{T}=$ control without lime; calcium carbonate at 10 and 20 1/ha doses (C10 and C20, respectively), magnesium oxide in 10 y $20 \mathrm{l} / \mathrm{ha}$ doses $(\mathrm{Mg} 10$ and $\mathrm{Mg} 20$, respectively), calcium carbonate + magnesium oxide mixed at $5+5$ and $10+10$ 1/ha doses of each one $(\mathrm{C} 5+\mathrm{Mg} 5$ and $\mathrm{C} 10$ $+\mathrm{Mg} 10$ respectively). Error bars as standard error. Different letters mean statistical differences between treatments (Tukey test, $\mathrm{p}<0,05$ ). significativas con respecto al testigo sin cal. Los tratamientos de solo óxido de magnesio y el testigo presentaron los valores más bajos de peso fresco de raíces y no hubo diferencias significativas entre ambos (Figura 3).

Con la variable peso seco, el tratamiento de Ca 10 presentó el valor más alto, aunque fue significativamente igual a la dosis de $20 \mathrm{l} / \mathrm{ha}$ y a los dos tratamientos de $\mathrm{Ca}+\mathrm{Mg}$. Los resultados mostraron que el carbonato de calcio solo o en mezcla con óxido de magnesio, aumentaron en forma significativa la biomasa seca radical del sorgo, y que la dosis de 10 l/ha fue el mejor tratamiento si se toma en cuenta el costo de su uso. Un efecto similar ocurrió con la biomasa aérea.

La aplicación de enmiendas neutralizó la acidez intercambiable y en la solución del suelo, disminuyendo el efecto fitotóxico del Al, y aumentó el contenido de $\mathrm{Ca}$ y/o $\mathrm{Mg}$ en el suelo. Estos efectos contribuyeron a mejorar el crecimiento de raíces, tal y como ha sido descrito por Rengel (1992).

El incremento de la materia seca del sistema radical en suelos ácidos se vio favorecido por la aplicación de enmiendas, tal y como ha sido descrito en un estudio donde encontraron un incremento en el crecimiento de raíces y una disminución en su mortalidad como consecuencia de la aplicación de cal en especies forestales (Matzner et al., 1986).

$\mathrm{Al}$ respecto, se han reportado incrementos en la longitud y densidad radical por parte de plantas de trigo y cebada, sembradas en suelos ácidos bajo tratamientos de encalado (Tang et al., 2003). Asimismo, se determinó que la aplicación de cal dolomita incrementó en un $66 \%$ el crecimiento de raíces en el cultivo del trigo sembrado en un Oxisol (Caires et al., 2006).

\section{Efecto de las enmiendas en la absorción de nutrimentos}

Hubo efecto significativo de la aplicación de enmiendas en la absorción de $\mathrm{Ca}, \mathrm{Mg}, \mathrm{K}, \mathrm{S}, \mathrm{Cu}$ y $\mathrm{Mn}$ en el sorgo (Cuadro 2), mientras que en el caso de $\mathrm{N}$, $\mathrm{P}, \mathrm{Fe}, \mathrm{Zn}$ y $\mathrm{B}$ no hubo diferencias significativas entre tratamientos. Los tratamientos de carbonato de calcio presentaron las mayores cantidades absorbidas de $\mathrm{Ca}, \mathrm{K}$ y $\mathrm{Mn}$, los cuales estadísticamente difieren del resto de tratamientos. Era de esperar que este material como fuente de $\mathrm{Ca}$ incrementara los contenidos de este 


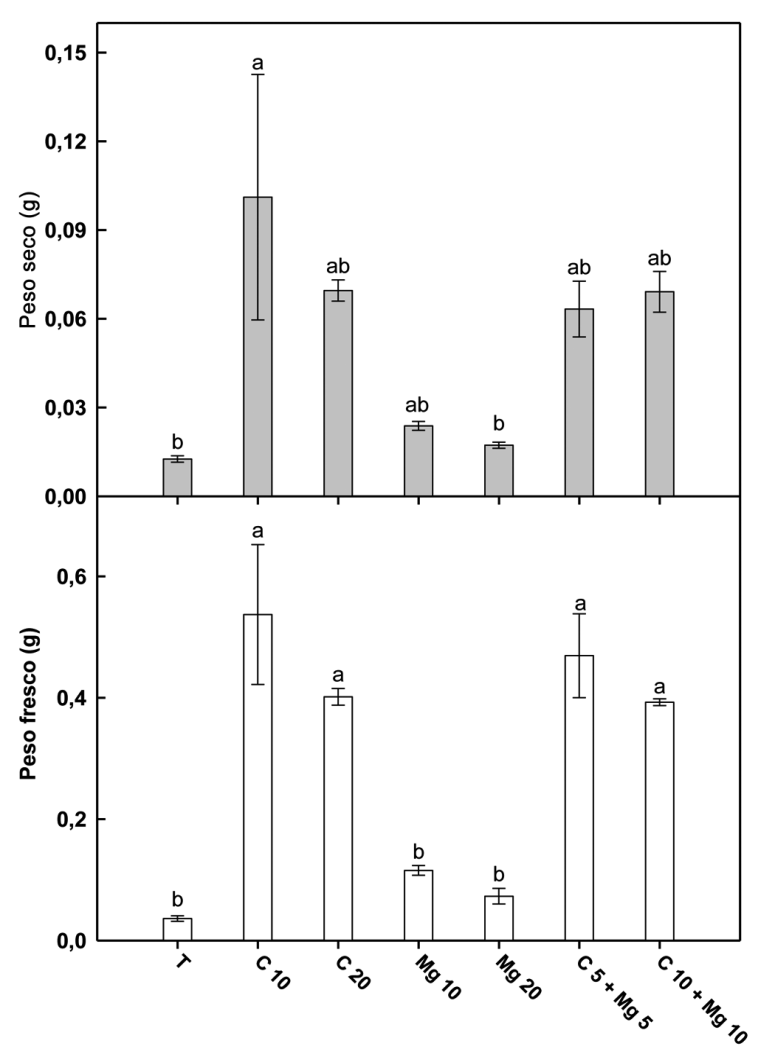

Tratamientos

Figura 3. Efecto de la aplicación de enmiendas líquidas sobre el peso fresco y seco de la raíz del sorgo sembrado en potes con un suelo Ultisol. San Pedro de Montes de Oca, San José, Costa Rica. 2011.

$\mathrm{T}=$ testigo sin enmienda; carbonato de calcio en dosis de 10 y 20 l/ha (C10 y C20 respectivamente), óxido de magnesio en dosis de 10 y 20 1/ha (Mg10 y Mg20 respectivamente), carbonato de calcio + óxido de magnesio mezclados en dosis de $5+5$ y $10+10$ l/ ha de cada uno $(\mathrm{C} 5+\mathrm{Mg} 5$ y $\mathrm{C} 10+\mathrm{Mg} 10$ respectivamente). Barras de error con base en error estándar. Letras diferentes implican diferencias estadísticas entre tratamientos, según la prueba de Tukey $(\mathrm{p}<0,05)$.

Figure 3. Effect of liquid liming amendments on dry and fresh weight of the root biomass of sorghum planted in pots with an Ultisol. San Pedro, Montes de Oca, San José, Costa Rica. 2011.

$\mathrm{T}=$ control without lime; calcium carbonate at 10 and $20 \mathrm{l} / \mathrm{ha}$ doses (C10 and C20, respectively), magnesium oxide at 10 y $20 \mathrm{l} / \mathrm{ha}$ doses $(\mathrm{Mg} 10$ and $\mathrm{Mg} 20$, respectively), calcium carbonate + magnesium oxide mixed in $5+5$ and $10+10$ 1/ha doses of each one (C5 + Mg5 and C10 $+\mathrm{Mg} 10$ respectively). Error bars as standard error. Different letters mean statistical differences between treatments (Tukey test, $\mathrm{p}<0,05$ ). nutrimento en el tejido vegetal y por lo tanto, también aumentara la absorción (Espinosa y Molina, 1999; Molina y Rojas, 2005). Al aumentar la producción de biomasa como consecuencia de la neutralización de la acidez y el suministro de $\mathrm{Ca}$, otros elementos como el $\mathrm{K}$ también fueron favorecidos en su absorción.

Hubo un incremento en la absorción de $\mathrm{Mg}$ y $\mathrm{Cu}$ en los tratamientos de $\mathrm{Ca}+\mathrm{Mg}$. El óxido de magnesio solo también incrementó la absorción de $\mathrm{Mg}$ en el sorgo. Este resultado se presentó debido a que es una fuente de $\mathrm{Mg}$. Resultados similares fueron encontrados al aplicar una enmienda con $\mathrm{Mg}$ en el cultivo de naranja (Molina y Rojas, 2005). La absorción de $\mathrm{S}$ también aumentó en los tratamientos con mayor producción de biomasa.

El tratamiento testigo presentó los valores más bajos de absorción de la mayoría de nutrimentos, principalmente $\mathrm{Ca}$ y $\mathrm{Mg}$. La baja producción de materia seca en el testigo fue una de las causas de su menor absorción de nutrimentos, esto fue consecuencia del efecto tóxico del Al. Baligar et al. (1993), encontraron una correlación negativa entre el $\mathrm{Al}$ en el suelo y la absorción de nutrimentos en la parte aérea. $\mathrm{Al}$ respecto, se reportó un incremento en el contenido de $\mathrm{Ca}, \mathrm{Mg}$ y $\mathrm{Mn}$ en la hoja bandera del arroz, debido a la aplicación de enmienda, y un incremento en la absorción de $\mathrm{K}$ y $\mathrm{S}$ en las hojas del frijol cuando se aplicó cal dolomita (Sorrato y Crusciol, 2008).

La aplicación de solo Ca afectó la absorción de $\mathrm{Mg}$. Al respecto, Juo y Uzu (1977) indican que en Ultisoles, la aplicación de enmiendas cálcicas como $\mathrm{CaCO}_{3}$ y $\mathrm{Ca}(\mathrm{OH})_{2}$ podría disminuir la absorción de $\mathrm{Mg}$ debido a un desbalance catiónico generado en el suelo $\mathrm{y}$ al incremento del $\mathrm{pH}$, el cual influencia la solubilidad del mismo. Se encontró respuesta en producción de materia seca con la aplicación de cal, pero la enmienda no tenía $\mathrm{Mg}$, por lo que el cultivo presentó deficiencias del elemento debido al antagonismo con $\mathrm{Ca}$ (Tan et al., 1992).

Ritche y Snuffer (2002), evaluaron la recuperación de una pastura mediante el uso de enmiendas cálcicas y dolomíticas, y su efecto en el contenido foliar de nutrimentos. Durante el proceso de recuperación, el contenido de $\mathrm{Ca}$ y $\mathrm{Mg}$ foliar fue proporcional a la saturación de estos elementos en el suelo, posterior a la aplicación de las enmiendas. 
Cuadro 2. Efecto de las enmiendas líquidas sobre la acumulación de $\mathrm{Ca}, \mathrm{Mg}, \mathrm{K}, \mathrm{S} \mathrm{Cu}$ y Mn en plantas de sorgo sembrado en potes con suelo Ultisol. San Pedro de Montes de Oca, Costa Rica. 2011. Carbonato de calcio en dosis de 10 y $201 /$ ha (Ca 10 y Ca 20 respectivamente), óxido de magnesio en dosis de 10 y 20 1/ha (Mg 10 y Mg 20 respectivamente), carbonato de calcio + óxido de magnesio mezclados en dosis de $5+5$ y $10+10$ 1/ha de cada uno $(\mathrm{Ca} 5+\mathrm{Mg} 5$ y Ca $10+\mathrm{Mg} 10$ respectivamente).

Table 2. Effect of liquid liming amendments on $\mathrm{Ca}, \mathrm{Mg}, \mathrm{K}, \mathrm{S}, \mathrm{Cu}$, and $\mathrm{Mn}$ accumulation on sorghum growth in pots of an Ultisol. San Pedro de Montes de Oca. 2011. Calcium carbonate at 10 and 20 1/ha doses (Ca 10 and Ca 20, respectively), magnesium oxide a 10 y 20 1/ha doses ( $\mathrm{Mg} 10$ and $\mathrm{Mg} 20$ respectively), Calcium carbonate + magnesium oxide mixed at $5+5$ and $10+10$ 1/ha doses of each one $(\mathrm{Ca} 5+\mathrm{Mg} 5$ and $\mathrm{C} 10+\mathrm{Mg} 10$ respectively).

\begin{tabular}{|c|c|c|c|c|c|c|c|c|c|c|c|c|}
\hline \multirow{2}{*}{$\begin{array}{c}\text { Tratamiento } \\
\mathrm{l} / \mathrm{ha}\end{array}$} & \multicolumn{2}{|r|}{$\mathbf{C a}$} & \multicolumn{2}{|c|}{$\mathbf{M g}$} & \multicolumn{2}{|c|}{$\mathbf{K}$} & \multicolumn{2}{|r|}{$\mathbf{S}$} & \multicolumn{2}{|c|}{$\mathrm{Cu}$} & \multicolumn{2}{|c|}{ Mn } \\
\hline & & & & & & & lanta & & & & & \\
\hline $\mathrm{T}$ & 0,012 & $\mathrm{c}^{*}$ & 0,02 & $\mathrm{~d}$ & 0,085 & $\mathrm{~b}$ & 0,009 & d & 0,0002 & $\mathrm{c}$ & 0,001 & $\mathrm{~cd}$ \\
\hline Ca 10 & 0,501 & $\mathrm{a}$ & 0,044 & d & 0,786 & $\mathrm{a}$ & 0,103 & a & 0,0006 & $a b$ & 0,005 & $a b$ \\
\hline Ca 20 & 0,501 & $\mathrm{a}$ & 0,046 & d & 0,736 & $\mathrm{a}$ & 0,082 & $\mathrm{~b}$ & 0,0005 & $\mathrm{bc}$ & 0,005 & $a b$ \\
\hline $\operatorname{Mg} 10$ & 0,013 & $\mathrm{c}$ & 0,202 & $\mathrm{c}$ & 0,418 & $\mathrm{~b}$ & 0,032 & $\mathrm{~cd}$ & 0,0003 & $\mathrm{c}$ & 0,003 & $\mathrm{~cd}$ \\
\hline $\operatorname{Mg} 20$ & 0,011 & $\mathrm{c}$ & 0,233 & $\mathrm{bc}$ & 0,323 & $\mathrm{~b}$ & 0,039 & $\mathrm{c}$ & 0,0003 & $\mathrm{c}$ & 0,002 & $\mathrm{c}$ \\
\hline $\mathrm{Ca} 5+\operatorname{Mg} 5$ & 0,228 & $\mathrm{~b}$ & 0,284 & $a b$ & 0,951 & $\mathrm{a}$ & 0,090 & $a b$ & 0,0008 & $\mathrm{a}$ & 0,006 & $\mathrm{a}$ \\
\hline $\mathrm{Ca} 10+\mathrm{Mg} 10$ & 0,277 & $\mathrm{~b}$ & 0,285 & $\mathrm{a}$ & 0,935 & $\mathrm{a}$ & 0,097 & $\mathrm{a}$ & 0,0008 & $\mathrm{a}$ & 0,004 & $\mathrm{bc}$ \\
\hline
\end{tabular}

* Letras diferentes a la derecha indican diferencias significativas $(\mathrm{p}<0,05)$ según la prueba de Tukey / Different letters mean statistical differences (Tukey test, $\mathrm{p}<0.05$ ).

\section{Efecto de las enmiendas en la fertilidad del suelo}

La aplicación de enmiendas mejoró la fertilidad del suelo al aumentar el $\mathrm{pH}$ y disminuir el contenido de acidez intercambiable y la saturación de acidez. El tratamiento de $\mathrm{Mg} 20$ presentó el mayor efecto en reducir la acidez del suelo e incrementar los contenidos de $\mathrm{Mg}$ y S (Cuadro 3) a los 45 días después de sembrado el sorgo. Los tratamientos de C $10 \mathrm{y}$ C 20 , y C $10+\mathrm{Mg} 10$, presentaron los valores más elevados de $\mathrm{Ca}$ con diferencias significativas con respecto a otros tratamientos.

El pH aumentó y el contenido de acidez intercambiable disminuyó con la aplicación de enmiendas, todos los tratamientos fueron superiores al testigo en forma significativa (Cuadro 3). El óxido de magnesio en dosis de 20 1/ha alcanzó el pH más alto y el nivel más bajo de acidez intercambiable con diferencias significativas con respecto al resto de tratamientos. $\mathrm{Al}$ aumentar la dosis de enmienda líquida se redujo el nivel de acidez intercambiable.

La saturación de acidez se redujo significativamente en los tratamientos de enmienda líquida con respecto al testigo. El tratamiento de $\mathrm{Mg} 20$ presentó la mayor reducción del porcentaje de saturación de acidez, significativamente distinto del resto de tratamientos (Cuadro 3). Este resultó en un 3,3\% de saturación de acidez en comparación con el testigo que tenía casi $95 \%$, traduciéndose en la neutralización de un 91\% de la saturación de acidez del suelo original, probablemente debido a su alto poder de neutralización de la acidez del suelo por ser un óxido de gran fineza. Así se ha indicado que el óxido de magnesio es la fuente con mayor efecto de neutralización de la acidez del suelo (Alcarde, 1992; Molina, 1998).

Por otro lado, ambos tratamientos de $\mathrm{Ca}+\mathrm{Mg}$ tuvieron un efecto significativo en la reducción del porcentaje de saturación de la acidez, logrando llevarla hasta valores entre $25 \%$ y $60 \%$, los cuales propician condiciones más favorables para un cultivo en comparación con el estado original del suelo.

El óxido de magnesio superó a los tratamientos de carbonato de calcio en la disminución de la acidez del suelo. El óxido de magnesio tiene un mayor equivalente químico que el carbonato de calcio que le permite un mayor poder de neutralización de la acidez del suelo (Molina, 1998). La aplicación de carbonato de calcio líquido aumentó el nivel de Ca en el suelo, en forma similar a lo que reportan otros investigadores con enmiendas sólidas (Furtini et al., 1999; Molina 
Cuadro 3. Efecto de las enmiendas líquidas en la fertilidad de un Ultisol. San Pedro de Montes de Oca, Costa Rica. 2011. Carbonato de calcio en dosis de 10 y 20 l/ha (C 10 y C 20 respectivamente), óxido de magnesio en dosis de 10 y 20 1/ha (Mg 10 y Mg 20 respectivamente), carbonato de calcio + óxido de magnesio mezclados en dosis de $5+5$ y $10+10$ l/ha de cada uno (C $5+\mathrm{Mg} 5$ y C $10+\mathrm{Mg} 10$ respectivamente).

Table 3. Effect of liquid liming amendments on fertility parameters of an Ultisol. San Pedro, Montes de Oca, Costa Rica. 2011. Calcium carbonate at 10 and 20 l/ha doses (C 10 and C 20, respectively), magnesium oxide at 10 y 20 l/ha doses (Mg 10 and $\mathrm{Mg}$ 20, respectively), calcium carbonate + magnesium oxide mixed at $5+5$ and $10+10$ 1/ha doses of each one (C 5 $+\operatorname{Mg} 5$ and $\mathrm{C} 10+\mathrm{Mg} 10$, respectively).

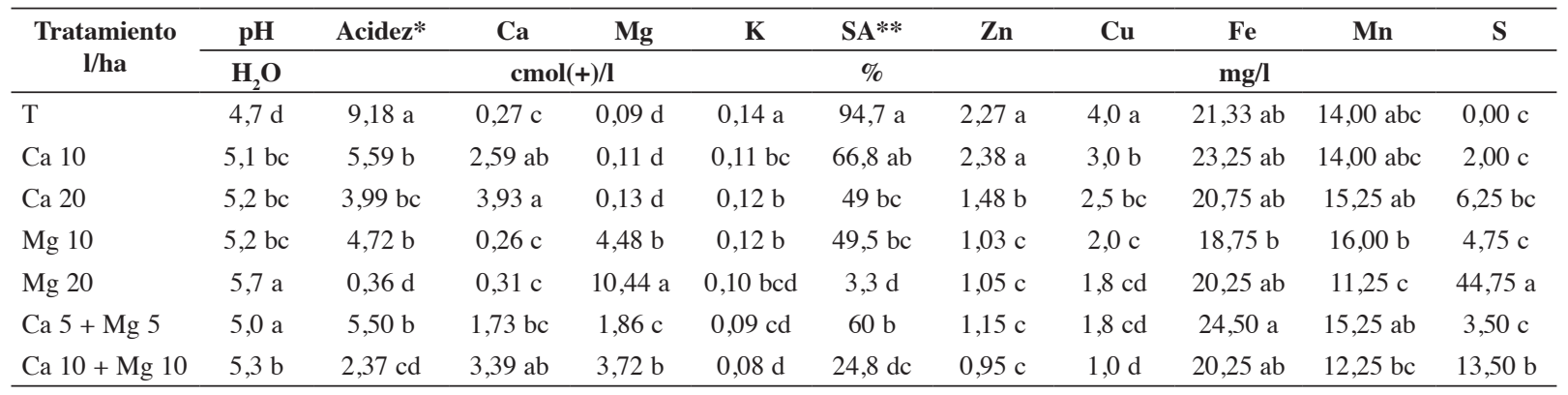

* Acidez intercambiable / Exchangeable acidity.

** Porcentaje de saturación de acidez / Percentage of acidity saturation.

Letras diferentes a la derecha implican diferencias estadísticas $(\mathrm{p}<0,05)$, según la prueba de Tukey / Different letters on the right mean statistical differences (Tukey test, $\mathrm{p}<0.05$ ).

y Rojas, 2005; Sorrato y Crusciol, 2008), quienes señalan incrementos en los contenidos de $\mathrm{Ca}$ en el suelo debido a la aplicación de enmiendas como el $\mathrm{CaCO}_{3}, \mathrm{MgCO}_{3}$, y la cal dolomítica. Ritche y Snuffer (2002), hallaron un incremento en el contenido de $\mathrm{Mg}$ en el suelo debido a la aplicación de fuentes de $\mathrm{Mg}$ como cal dolomítica o MgO. Un efecto similar fue encontrado con el uso de una mezcla de $\mathrm{CaCO}_{3}$ y $\mathrm{MgO}$ en Ultisol cultivado de naranja (Molina y Rojas, 2005); mientras que Salas et al. (1996) encontraron respuesta a la aplicación de 4 1/ha de cal dolomita en tiquisque blanco y un incremento significativo en los contenidos de $\mathrm{Ca}$ y $\mathrm{Mg}$ en el suelo. Como consecuencia del incremento en los contenidos de $\mathrm{Ca}$ y $\mathrm{Mg}$, la capacidad de intercambio catiónica efectiva (CICE) del suelo también aumentó.

El caso del $\mathrm{K}$, el tratamiento testigo presentó el mayor contenido, con diferencias significativas en comparación con los otros tratamientos (Cuadro 3). La aplicación de enmiendas líquidas disminuyó ligeramente la concentración de $\mathrm{K}$, probablemente debido al efecto antagónico que causaron el $\mathrm{Ca}$ y $\mathrm{Mg}$ (Bertsch, 1995).

Los contenidos de $\mathrm{Zn}$ y $\mathrm{Cu}$ disminuyeron con la aplicación de enmiendas líquidas (Cuadro 3), el efecto más significativo se obtuvo con los tratamientos de $\mathrm{Mg}$ y de $\mathrm{Ca}+\mathrm{Mg}$, probablemente debido al incremento en el pH que disminuyó su disponibilidad, y a la reacción química directa de las enmiendas con estos dos micronutrimentos que pudo formar compuestos insolubles. Con $\mathrm{Fe}$ y $\mathrm{Mn}$ no hubo un cambio significativo en sus contenidos, quizás porque sus niveles no eran altos en este suelo, contrario a lo que generalmente se esperaría de un Ultisol fuertemente ácido como el del presente estudio.

Las enmiendas líquidas neutralizaron la acidez intercambiable casi de forma inmediata después de su aplicación (Figura 4), con una disminución de sus valores en todos los tratamientos de cal hasta el día dieciocho después de la aplicación. A partir de ese momento, los valores de $\mathrm{Al}$ intercambiable empezaron a restituirse en todos los tratamientos, con excepción de $\mathrm{Mg} 20$ que mantuvo casi el mismo contenido bajo de acidez desde el inicio, probablemente por efecto de la dosis que era muy alta y de su mayor poder de neutralización. El efecto residual de la mayoría de tratamientos fue disminuyendo con el tiempo, y al día 44 cuando finalizó el experimento, las enmiendas líquidas neutralizaron entre 40 y $96 \%$ de la acidez intercambiable presente en el suelo (Figura 4). Los 


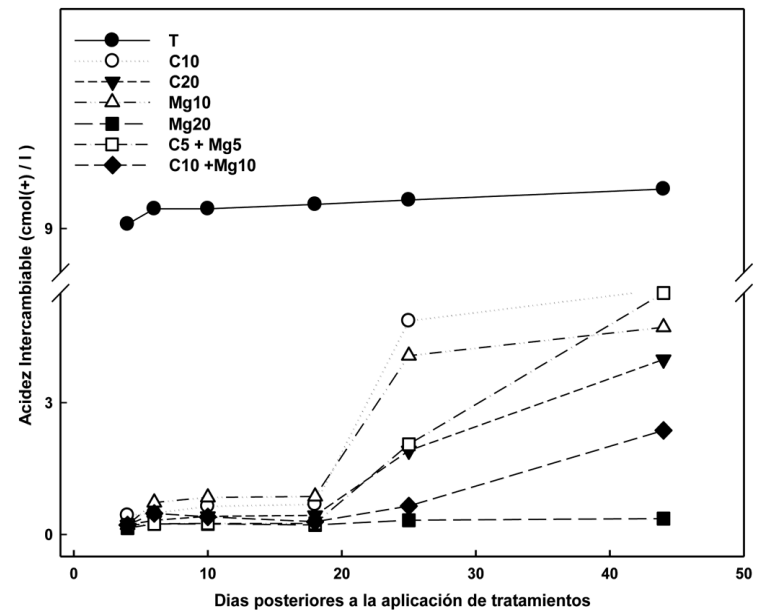

Figura 4. Efecto de la aplicación de cales líquidas sobre la acidez intercambiable de un suelo Ultisol en función del tiempo posterior a la aplicación. San Pedro de Montes de Oca, San José, Costa Rica. 2011.

$\mathrm{T}=$ testigo sin enmienda; carbonato de calcio en dosis de 10 y 20 1/ha (C10 y C20 respectivamente), óxido de magnesio en dosis de 10 y 20 1/ha (Mg10 y Mg20 respectivamente), carbonato de calcio + óxido de magnesio mezclados en dosis de $5+5$ y $10+101 /$ ha de cada uno (C5 + Mg5 y C10 + Mg10 respectivamente).

Figure 4. Effect of liquid liming on soil exchange acidity in an Ultisol soil related to time after treatment application. San Pedro, Montes de Oca, San José, Costa Rica. 2011.

$\mathrm{T}=$ control without lime;calcium carbonate at 10 and 20 1/ha doses (C10 and C20, respectively), magnesium oxide at 10 y 20 1/ha doses $(\mathrm{Mg} 10$ and Mg20, respectively), calcium carbonate + magnesium oxide mixed at $5+5$ and $10+10$ 1/ha doses of each one $(\mathrm{C} 5+\mathrm{Mg} 5$ and $\mathrm{C} 10+\mathrm{Mg} 10$, respectively).

tratamientos de $\mathrm{C} 10$ y $\mathrm{C} 5+\mathrm{Mg} 5$, con la mejor producción de biomasa, presentaron valores bajos de acidez intercambiable (menos de $1 \mathrm{cmol}(+) / \mathrm{l}$ ) durante los primeros dieciocho días después de aplicados; posteriormente parte de la acidez empezó a restituirse después de los veinticinco días. A los 44 días, la acidez intercambiable en ambos tratamientos alcanzó valores de $5,5 \mathrm{cmol}(+) / 1$ y la saturación de acidez fue de $60 \%$ o más. Estos resultados mostraron que las enmiendas líquidas fueron de reacción muy rápida, pero a las dosis utilizadas en este estudio su efecto residual fue de corto plazo.

El pH del suelo se incrementó drásticamente durante los primeros seis días posteriores a la aplicación de los tratamientos (Figura 5); sin embargo, a partir de ese momento comenzó a descender hasta valores cercanos al inicial. El tratamiento de $\mathrm{Mg} 20$ presentó también esta tendencia a un incremento drástico, y un descenso gradual, pero, al finalizar el experimento a los 44 días, el $\mathrm{pH}$ reportado para dicho tratamiento fue el más alto de todos y estadísticamente distinto al resto de tratamientos (Cuadro 3), superando el valor inicial por un margen mayor que los demás tratamientos (Figura 5).

En vista de que las enmiendas líquidas se mezclaron bien con el suelo antes de sembrar y que no

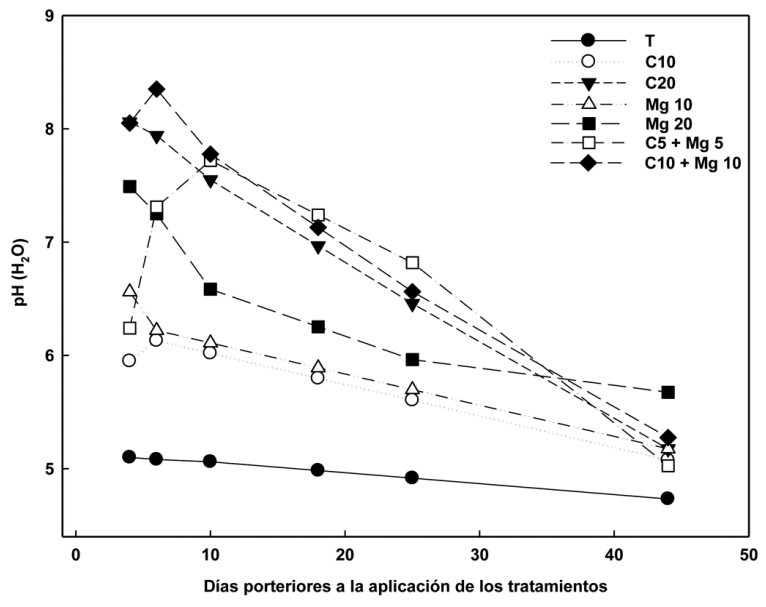

Figura 5. Efecto de la aplicación de enmiendas líquidas sobre el ph de un suelo Ultisol en función del tiempo posterior a la aplicación. San Pedro de Montes de Oca, San José, Costa Rica. 2011.

$\mathrm{T}=$ testigo $\sin$ enmienda; carbonato de calcio en dosis de 10 y 20 1/ha (C10 y C20 respectivamente), óxido de magnesio en dosis de 10 y 20 1/ha (Mg10 y Mg20 respectivamente), carbonato de calcio + óxido de magnesio mezclados en dosis de $5+5 \mathrm{y}$ $10+10$ 1/ha de cada uno $(\mathrm{C} 5+\mathrm{Mg} 5$ y C10 $+\mathrm{Mg} 10$ respectivamente).

Figure 5. Effect of liquid liming amendments on ph in an Ultisol, related to time after treatment application. San Pedro, Montes de Oca, San José, Costa Rica. 2011.

$\mathrm{T}=$ control without lime; calcium carbonate at 10 and 20 1/ha doses (C10 and C20, respectively), magnesium oxide at 10 y $20 \mathrm{l} / \mathrm{ha}$ doses $(\mathrm{Mg} 10$ and $\mathrm{Mg} 20$, respectively), calcium carbonate + magnesium oxide mixed at $5+5$ and $10+101 /$ ha doses of each one $(\mathrm{C} 5+\mathrm{Mg} 5$ and $\mathrm{C} 10+\mathrm{Mg} 10$, respectively. 
quedó evidencia visual de residuos de los productos sin reaccionar, se asumió que las enmiendas reaccionaron completamente desde el inicio de su aplicación, y por tal motivo se obtuvieron incrementos fuertes del $\mathrm{pH}$ apenas unos días después de su aplicación, contrario a lo que se esperaría de una enmienda sólida. Estos resultados mostraron que la enmienda disuelta en agua y con una granulometría tan fina fue capaz de reaccionar en un lapso muy corto.

El efecto rápido de neutralizar la acidez del suelo de las enmiendas líquidas estuvo relacionado con la naturaleza química y física de la fuente. El carbonato de calcio utilizado fue micronizado y contuvo partículas de malla 625 mesh, que le permitió tener una reacción química casi inmediata en el suelo debido a su alto grado de fineza. El óxido de magnesio utilizado también fue micronizado, siendo la fuente de enmienda con el mayor poder de neutralización de la acidez del suelo (Alcarde, 1992; Molina, 1998; Espinosa y Molina, 1999).

El descenso del pH (Figura 5) a lo largo del experimento, estuvo relacionado con las características químicas y mineralógicas de los suelos ácidos del tópico. Debido a la alta capacidad buffer presente en dichos suelos, estos pueden resistir cambios fuertes de $\mathrm{pH}$ aun cuando se adicionen dosis muy fuertes de una enmienda (Alvarado et al., 2010). Esto se debe a la mineralogía de las arcillas, las cuales frecuentemente corresponden a sistemas de óxidos o bien amorfos u aluminosilicatos con revestimientos de óxidos.

\section{AGRADECIMIENTOS}

Al Dr. Víctor Jiménez por su colaboración en la estadística de los datos, así como su ayuda en la elaboración y revisión del presente trabajo. A la M.Sc. María Viñas por su colaboración en la determinación de las variables de crecimiento vegetal, y su apoyo a lo largo del ensayo.

A la compañía Agronegocios Suplidora Verde por la donación de las enmiendas empleadas en el presente trabajo.

\section{LITERATURA CITADA}

Alcarde, J.C. 1992. Corretivos da acidez dos solos: características e interpretacoes técnicas. Boletin Técnico No. 6, ANDA, BRA.

Alvarado, A., E. Molina, y G. Cabalceta. 2010. Acidez y encalado de suelos. En: A. Usón Murillo et al., editores, Tecnología de suelos: estudios de casos. Ediciones de la Universidad de Lleida, Zaragoza, ESP. p. 69-99.

Alvarado, A., y J.L. Fallas. 2004. La saturación de acidez y el encalado sobre el crecimiento de la teca (Tectona grandis l.f.) en suelos ácidos de Costa Rica. Agron. Costarricense 28(1):81-87.

Baligar, B., R. Schaffert, H. Dos Santos, G. Pitta, and A. Bahia. 1993. Soil aluminum effects on uptake, influx, and transport of nutrients in sorghum genotypes. Plant Soil 150:271-277.

Barber, S. 1984. Liming materials and practices. In: F. Adams, editor, Soil acidity and liming. ASA, WI, USA. p. 171-209.

Bertsch, F. 1995. La fertilidad de los suelos y su manejo. Asociación Costarricense de la Ciencia del Suelo, San José, CRC.

Brown, T., R. Koenig, D. Huggins, R. Harsh, and R. Rossi. 2008. Lime effects on soil acidity, crop yield, and aluminum chemistry in direct-seeded cropping systems Soil Sci. Soc. Am. J. 72:634-640.

Cabalceta, G., y E. Molina. 2006. Niveles críticos de nutrimentos en suelos de Costa Rica utilizando la solución extractora Mehlich 3. Agron. Costarricense 30(2):31-44.

Caires, E., J. Leal, S. Churka, G. Barth, and F. Garbuio. 2006. Surface application of lime ameliorates subsoil acidity and improves root growth and yield of wheat in an acid soil under no-till system. Sci. Agric. 63:502-509.

Curtin, D., and J.K. Syers. 2002. Lime-induced changes in indices of soil phosphate availability. Soil Sci. Soc. Am. J. 65:147-152.

Díaz-Romeu, R., y A. Hunter. 1978. Metodología de muestreo de suelos, análisis químico de suelos y tejido vegetal e investigación en invernadero. CATIE, Turrialba, CRC.

Edmeades, D., and A. Ridley. 2003. Using lime to ameloriate topsoil and subsoil acidity. In: Z. Rengel, editor, 
Handbook of soil acidity. Marcel Dekker Inc. NY, USA. p. 297-336.

Espinosa, J., y E. Molina. 1999. Acidez y encalado de los suelos. INPOFOS, Quito, ECU.

Furtini, A.E., A. Vilela, F. Ribeiro, and I. Ribeiro. 1999. Liming effects on growth of native woody species from brazilian savannah. Pesq. Agropec. Bras. 34:829837.

Guevara, E., y V. Jiménez. 1998. Principios y aplicaciones de la fisiología vegetal. Editorial de la Universidad de Costa Rica, San José, CRC.

Juo, A., and F. Uzu. 1977. Liming and nutrient interactions in two ultisols from southern Nigeria. Plant Soil 47:419-430.

Kamprath, E.J. 1984. Crop response to lime on soils in the tropics. En: F. Adams, editor, Soil acidity and liming, ASA, WI, USA. p. 349-369.

Matzner, E., D. Murac, and H. Fortmann. 1986. Soil acidity and its relationship to root growth in declining forest stands in Germany. Water Air Soil Pollut. 31:273-282.

Mata, R., A. Vázquez, A. Rosales, y D. Salazar. 2012. Mapa digital de suelos de Costa Rica. Asociación Costarricense de la Ciencia del Suelo, San José, CRC. Escala 1:200000.

Molina, E. 1998. Encalado para la corrección de la acidez del suelo. ACSS, San José, CRC.

Molina, E. 2001. Manejo de la acidez y encalado de los suelos. En: G. Meléndez, y E. Molina, editores, Memorias Seminario de suelos y manejo de la nutrición de cultivos en Costa Rica, Laboratorio de Suelos, CIA-UCR, San José, CRC. p. 28-41.

Molina, E., y E. Bornemisza. 2006. Nivel crítico de zinc en suelos de Costa Rica. Agron. Costarricense 30(2):45-59.

Molina, E., y A. Rojas. 2005. Efecto del encalado en el cultivo de naranja valencia en la Zona Norte de Costa Rica. Agron. Costarricense 29(3):81-95.

Peters, J.B., K.A. Kelling, and E.E. Schulte. 1996. Choosing between liming materials. Extension Cooperative Publication A3671, University of Wisconsin, Madison, WI, USA.
Rengel, Z. 1992. Role of calcium in aluminum toxicity. New Phytol. 121:499-513.

Ritchey, K.D., and D. Snuffer. 2002. Limestone, gypsum, and magnesium oxide influence on restoration of an abandoned Appalachian pasture. Agron. J. 94:830-839.

Salas, R., E. Molina, and D. Bouldin. 1996. Lime response on tanier in an Ultisol of Costa Rica. Commun. Soil Sci. Plant Anal. 27:2477-2484.

Salas, R., T. Smyth, D. Alpizar, J. Boniche, A. Alvarado, y A. Rivera. 2002. Corrección de la acidez del suelo con Ca y Mg y su efecto en el desarrollo del sistema radical del palmito en la etapa de previvero. Agron. Costarricense 26(2):87-94.

Salisbury, F., y C. Ross. 1999. Fisiología vegetal. Editorial Iberoamericana, MEX.

Sidari, M., M. Panuccio, and A. Muscolo. 2004. Influence of acidity on growth and biochemistry of Pennisetum clandestinum. Biol. Plant. 48:133-136.

Soratto, P., and C. Crusciol. 2008. Dolomite and phosphogypsum surface application effects on annual crops nutrition and yield. Agron. J. 100:261-270.

Taiz, L., and E. Zeiger. 2002. Plant physiology. 3 ed. Sinauer Associates, Sunderland, GBR.

Tan, K., W. Keltjens, and G. Findenegg. 1992. Acid soil damage in sorghum genotypes: role of magnesium deficiency and root impairment. Plant Soil 139:149155 .

Tang, C., E. Diatloff, Z. Rengel, and B. McGann. 2001. Growth response to subsurface soil acidity of wheat genotypes differing in aluminum tolerance. Plant Soil 236:1-10.

Tang, C., Z. Rengel, E. Diatloff, and C. Gazey. 2003. Responses of wheat and barley to liming on a sandy soil with subsoil acidity. Field Crop Res. 80:235-244.

Valerio, J.M., y E. Molina. 2012. Evaluación de una fuente de enmienda líquida en el rendimiento de arroz en un Ultisol de la Zona Norte de Costa Rica. Agron. Costarricense 36(1):89-96. 
